\title{
HELIO- AND ASTEROSEISMOLOGY
}

\author{
Chairperson and Editor: W. DÄPPEN
}




\title{
HELIO- AND ASTEROSEISMOLOGY
}

\author{
W. DÄPPEN \\ University of Southern California \\ Los Angeles, CA 90089-1342, USA
}

\section{Introduction}

Since the early 1960 s the surface of the Sun has been know to be in a regular pulsating motion with periods of about 5 minutes. While at the beginning various explanations were offered, only in the 1970s it was recognized that these so-called solar oscillations are manifestations of global motions of the Sun about its equilibrium. Helioseismology is the name of the branch of astrophysics that deals with deciphering these data, that cover the whole range of spherical harmonics from $l=0$ (radial) to very high angular order (above $l=1000$ ). Thanks to observational data of superb quality (each of the oscillation frequencies is measured accurately to better than one part in ten thousand), our knowledge of the Sun has leap-frogged in the last 20 years. For instance, we now know the run of temperature inside the Sun, or have good information about the internal solar rotation. In the solar neutrino problem the data from solar oscillations have become a compulsory testing stone for any model proposed to explain the discrepancy between observed and theoretically predicted solar neutrinos.

Asteroseismology is the hope to extend helioseismic techniques to stars. What has so far emerged? Nothing really analogous to solar oscillations, although several groups have tried to find them. However, there is progress, reported here. The principal difficulty comes from the fact that stars are so far away that they are not spatially resolved. Unless one resorts to tricks, such as traveling bumps in spectral lines, the star only reveals the lowest-l modes. Now, of course, the smaller the number of frequencies is, the less we can infer about the stellar interiors. In other words, the analogy to helioseismology is weaker. In this situation it is wise to look at the existing multi-periodic variable stars to gather experience. Such multi-mode variable stars do indeed exist. Essentially there are three classes of such stars: (i) 
Pulsating White dwarfs, (ii) Rotating Ap stars, (iii) $\delta$ Scuti stars. Although the aforementioned objects already marginally qualify for asteroseismology (in the eyes of some at least), there is a tremendous interest to see oscillations of solar-like stars. One of the highlights of this meeting was therefore the report on the first firm evidence for the detection of such oscillations (Kieldsen \& Bedding, section 12).

\section{Observations (E.J. Rhodes, Jr.)}

The field of observational helioseismology began in 1960 when Leighton, Noyes, and Simon (1962) discovered the so-called solar "5-minute" oscillations at the $\mathrm{Mt}$. Wilson Observatory. However, it was not until roughly two decades ago, when these 5-minute oscillations were demonstrated to be sound, or acoustic waves, which were trapped within the solar interior (Deubner, 1975), that observational helioseismology began to grow in earnest. In the nearly two decades which have elapsed since its infancy, observational helioseismology has been the source of many surprising new results concerning the solar interior - far more results than there is space to list here. These past two decades have also been a time of great intellectual ferment which has led to the development of new instrumental tools and new analysis techniques at an astounding rate, with many of these tools and techniques not even being contemplated two decades ago.

Today, however, helioseismology has outgrown its infancy. It has largely (although not entirely, as we shall see in a moment) moved from its early discovery stage, in which major new results concerning the solar interior were presented at nearly every international meeting in the field, into an extended period of consolidation in which some of its earliest successes are being only marginally refined, in which at some meetings more new analysis techniques are being announced than are new results, and in which the ever-lengthening temporal baseline of existing helioseismic observations is allowing unmistakable discoveries to be made of the first solar cycle dependencies of some of the Sun's helioseismic properties.

On the other hand, observational helioseismology has also reached the point where it is now being carried out by so many different teams of observers with so many different instruments that contradictory results have begun to appear in the literature in some areas within the field. And finally, with several new ground-based networks of imaging instruments soon to go into regular operation, and with several new space-based helioseismic instruments set to be launched on what is hoped to be an extended study of solar oscillations from space, observational helioseismology is also a field in which basic observational data will soon be multiplying much more rapidly than will the number of its available practitioners. 